\title{
EDITORIAL
}

\section{Stress and severity of illness in very low birth weight infants: what are we measuring?}

\section{Journal of Perinatology (2010) 30, 503-504; doi:10.1038/jp.2010.77}

Understanding the role of cortisol in response to stress and the subsequent modulation of inflammation in the very low birth weight (VLBW) infant population presents a significant challenge for neonatologists. Miletin et al. ${ }^{1}$ evaluated the contribution of cortisol concentrations in interpreting the severity of illness in this high-risk population. Early cortisol concentrations $(12-24 \mathrm{~h}$ of life) were obtained, and compared with a variety of measures of severity of illness, including CRIB II, SNAPPE-II and NEOMOD, as well as the clinical measures of perfusion assessed by echocardiography: mean blood pressure and superior vena cava (SVC) flow. A significant correlation was found between cortisol values and NEOMOD scores, but with none of the other measures. The authors concluded that VLBW infants mount a cortisol response in the face of organ dysfunction.

Measuring the severity of illness, particularly in the initial days of life, can provide neonatologists with critical information to assist in guiding therapies and counseling families. Both $\mathrm{CRIB}^{2}$ and SNAPPE $^{3}$ scores identify a variety of risk factors that are known to be predictive of mortality and adverse neurodevelopmental outcome. The NEOMOD score provides a measure of multiorgan dysfunction and has been shown to correlate with mortality when measured daily over the first 28 days. ${ }^{4,5}$ Severity of illness scores can be limited by the timing of the measurement. Although elevated scores in the initial days of life can be helpful in identifying those infants who are at a high risk of adverse outcome, those infants with less severity of illness initially may have poor late outcome related to subsequent confounding events such as late-onset sepsis or necrotizing enterocolitis. The NEOMOD score has the advantage of a continuous evaluation over 28 days to account for some of these late events, but may be less helpful early in the life of the infant.

Miletin examined the use of additional markers of severity of illness that may assist in the acute identification of those infants who are at the highest risk for adverse outcome. ${ }^{1}$ Cortisol values have been examined by a number of investigators in an attempt to identify infants with relative adrenal insufficiency. As these infants are at high risk for hypotension, echocardiographic measures were used to identify cardiovascular instability, which may be a component of relative adrenal insufficiency. ${ }^{6}$ No correlation was found between early cortisol concentrations and mean blood pressure or SVC flow. This is consistent with other studies that have not found an association between early cortisol concentrations and blood pressure or the need for inotropic support. ${ }^{7,8}$

The interpretation of cortisol values in VLBW infants has remained a challenge. In utero, cortisol concentrations are very low and can remain low in healthy preterm infants without demonstrating evidence of relative adrenal insufficiency. ${ }^{9,10}$ Other infants will have low cortisol concentrations in the face of significant cardiorespiratory illness, which has been associated with an increased risk of developing bronchopulmonary dysplasia. ${ }^{11-14}$ Despite this, prophylactic supplementation with hydrocortisone in high-risk infants did not reduce the incidence of bronchopulmonary dysplasia. ${ }^{15}$ Low cortisol concentrations at day 7 of life, rather than day 1 , have been associated with low blood pressure and the need for vasopressor agents, ${ }^{8}$ although this was not shown by others, ${ }^{7}$ and prophylactic treatment with hydrocortisone did not impact the need for vasopressor therapy. ${ }^{15}$ The clinical implication of a low cortisol value, at either day 1 or day 7 of life, remains unclear. The long-term outcome of infants with low cortisol concentrations is not adversely impacted. ${ }^{16}$

There is a growing concern regarding the clinical implication of high cortisol values in the VLBW population. ${ }^{1,7,15,16}$ The subset of critically ill infants who show an exaggerated response to stress has been associated with increased adverse outcomes, specifically spontaneous gastrointestinal perforations, ${ }^{15}$ intraventricular hemorrhage, periventricular leukomalacia and death. ${ }^{16}$ These adverse outcomes are not associated with the use of hydrocortisone, rather with the innate response of these infants. Interestingly, the NEOMOD score, which evaluates organ dysfunction, was the only marker of severity of illness that correlated with elevated cortisol concentrations. ${ }^{1}$

The growing body of information suggests that measuring cortisol concentrations is not helpful in identifying infants with relative adrenal insufficiency that would benefit from supplementation with hydrocortisone. Clinical symptoms, with lack of response to vasopressor therapy, continue to be the indication for the need for treatment with hydrocortisone, regardless of whether the cortisol value is high or low. Low cortisol values in the absence of clinical symptoms are not associated with adverse outcomes. High cortisol values, in contrast, identify a vulnerable group of infants who are at high risk for a variety of adverse outcomes. What remains unclear is the mechanism of this stress response, 
and the source of the stress that is initiating it. This association with a measure of organ dysfunction may help to direct further investigation into this association, and provide insight on how to address alternative approaches to improve the outcome in this unique population.

\section{Conflict of interest}

The author declares no conflict of interest.

SW Aucott

Department of Pediatrics - Division of Neonatology, The Johns Hopkins University, Baltimore, MD, USA E-mail: saucott1@jbmi.edu

\section{References}

1 Miletin J, Pichova K, Doyle S, Dempsey EM. Serum cortisol values, superior vena cava flow and illness severity scores on very low birth weight infants. J Perinatol 2010; 30: $522-526$.

2 Parry G, Tucker J, Tarnow-Mordi W. CRIB II: an update of the clinical risk index for babies score. Lancet 2003; 361(9371): 1789-1791.

3 Richardson DK, Corcoran JD, Escobar GJ, Lee SK. SNAP-II and SNAPPE-II: Simplified newborn illness severity and mortality risk scores. J Pediatr 2001; 138(1): 92-100.

4 Janota J, Stranak Z, Statecna B, Dohnalova A, Sipek A, Simak J. Characterization of multiple organ dysfunction syndrome in very low birthweight infants: a new sequential scoring system. Shock 2001; 15(5): 348-352.

5 Janota J, Simak J, Stranak Z, Matthews T, Clarke T, Corcoran D. Critically ill newborns with multiple organ dysfunction: assessment by NEOMOD score in a tertiary NICU. Ir J Med Sci 2008; 177(1): 11-17.
6 Watterberg KL. Adrenal insufficiency and cardiac dysfunction in the preterm infant Pediatr Res 2002; 51(4): 422-424.

7 Aucott SW, Watterberg KL, Shaffer ML, Donohue PK. Do cortisol concentrations predict short-term outcomes in extremely low birth weight infants? Pediatrics 2008; 122(4): $775-781$.

8 Ng PC, Lee CH, Lam CW, Ma KC, Fok TF, Chan IH et al. Transient adrenocortical insufficiency of prematurity and systemic hypotension in very low birthweight infants. Arch Dis Child Fetal Neonatal Ed 2004; 89(2): F119-F126.

9 al Saedi S, Dean H, Dent W, Cronin C. Reference ranges for serum cortisol and 17-hydroxyprogesterone levels in preterm infants. J Pediatr 1995; 126(6): 985-987.

10 Soliman AT, Taman KH, Rizk MM, Nasr IS, Alrimawy H, Hamido MS. Circulating adrenocorticotropic hormone (ACTH) and cortisol concentrations in normal, appropriate-for-gestational-age newborns versus those with sepsis and respiratory distress: cortisol response to low-dose and standard-dose ACTH tests. Metabolism 2004; 53(2): 209-214.

11 Watterberg KL, Scott SM. Evidence of early adrenal insufficiency in babies who develop bronchopulmonary dysplasia. Pediatrics 1995; 95(1): 120-125.

12 Watterberg KL, Scott SM, Backstrom C, Gifford KL, Cook KL. Links between early adrenal function and respiratory outcome in preterm infants: airway inflammation and patent ductus arteriosus. Pediatrics 2000; 105(2): 320-324.

13 Scott SM, Cimino DF. Evidence for developmental hypopituitarism in ill preterm infants. J Perinatol 2004; 24(7): 429-434.

14 Peltoniemi 0, Kari MA, Heinonen K, Saarela T, Nikolajev K, Andersson S et al. Pretreatment cortisol values may predict responses to hydrocortisone administration for the prevention of bronchopulmonary dysplasia in high-risk infants. J Pediatr 2005; 146(5): 632-637.

15 Watterberg KL, Gerdes JS, Cole CH, Aucott SW, Thilo EH, Mammel MC et al. Prophylaxis of early adrenal insufficiency to prevent bronchopulmonary dysplasia: a multicenter trial. Pediatrics 2004; 114(6): 1649-1657.

16 Aucott SW, Watterberg KL, Shaffer ML and Donohue PK, for the PROPHET study group. Early cortisol values and long-term outcomes in extremely low birth weight infants. J Perinatol 2010; 30: 484-488. 